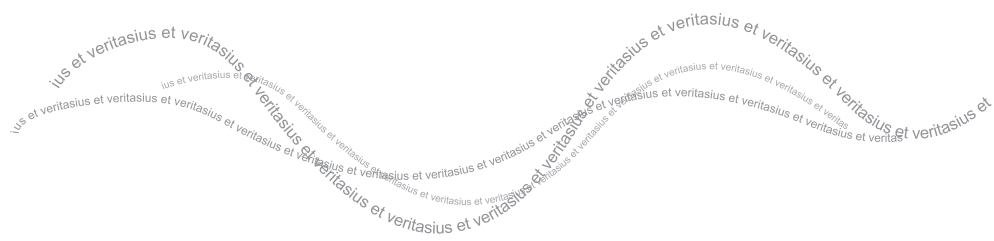

\title{
Relaciones de duración y divisibilidad del reglamento contractual: apuntes para una reconceptualización( ${ }^{(*)}(*+)$
}

\section{Long-term contracts and divisibility of contractual regulation: notes for a reconceptualization}

\section{LA CATEGORÍA DE LAS OBLIGACIONES DE DURACIÓN ES, POR ALGUNOS ASPECTOS, MÁS RESTRINGIDADE LO QUE LA OPINIÓN TRADICIONAL DEJA ENTENDER PORQUE, COMO HEMOS OBSERVADO ANTERIORMENTE, LA MERA REITERACIÓN DE LAS PRESTACIONES DURANTE EL TIEMPO NO ES POR SII MISMA DIRIMENTE PARA CALIFICARA LA RELACIÓN COMO DE DURACIÓN A FALTA DE SUFICIENTE AUTONOMÍA FUNCIONAL DE LAS PRESTACIONES INDIVIDUALES.}

\begin{abstract}
Resumen: El presente estudio analiza la función que cumple el tiempo en las transacciones contractuales específicas, con el fin de revisar la clásica categoría de los contratos de duración. En esta perspectiva, al considerar la diversidad de los intereses implicados, el autor reanaliza el tradicional concepto estructuralista de las obligaciones de duración referida a una prestación continuada y repetida, teniendo por objetivo asociar, principalmente, a los contratos de duración con la divisibilidad funcional del propio contrato, con la causa concreta realizada por la negociación a través del tiempo y con una consideración dinámica del interés que las partes pretenden satisfacer a través de la duración. Por tanto, la idea principal del análisis propuesto es que solo una atenta investigación del interés contractual de las partes, dejando de lado todo enfoque abstracto y tipológico, puede ofrecer una forma convincente de solucionar los problemas sobre la aún discutida naturaleza de los contratos de duración.
\end{abstract}

$\left(^{*}\right.$ Abogado por la Universidad de Bari "Aldo Moro", Italia. Especialista en Derecho Civil por la Universidad de Bri "Aldo Moro". Doctor por la Universidad de Sannio. Es Profesor e Investigador de Derecho Privado en la Universidad de Roma Tre. Miembro de la Sociedad Italiana de Estudiosos del Derecho Civil. Correo electrónico: francesco.longobucco@uniroma3.it

${ }^{* *}$ El título original del artículo es Rapporti di durata e divisibilità del regolamento contracttuale: Spunti per una riconcettualizzazione, que fue publicada originalmente en Archivio giuridico Filippo Serafini, vol. CCXXXV, 2015, fasc. 3, pág. 401-422. La traducción del italiano, autorizado por el autor, estuvo a cargo de César E. Moreno More, Adjunto de docencia en la Universidad Nacional Mayor de San Marcos a nivel de pre y posgrado, candidato a la Maestría con mención en Derecho Civil y Comercial por la misma casa de estudios.

${ }^{(* * *}$ Nota del Editor: este artículo fue recibido el 15 de mayo de 2017 y su publicación fue aprobada el 10 de junio 2017. 


\section{Relaciones de duración y divisibilidad del reglamento contractual: apuntes para una reconceptualización Long-term contracts and divisibility of contractual regulation: notes for a reconceptualization}

Palabras Clave: Contrato de Duración - Divisibilidad Funcional de la Relación -Causa Concreta de Duración - Meritoriedad Dinámica de los Intereses - Duración como Categoría Aplicativa

\begin{abstract}
The essay analyzes the role that time plays in the single specific transaction, in order to revisit the classic category of long-term contracts. In this perspective, considering the variety of interests involved, the author reexamines the structuralist traditional concept of durable obligations related to a continuing and repeated prevision, aiming to associate long-term contracts mainly to the functional divisibility of the whole contract itself, to the concrete cause realized by negotiation through the time, to a dynamic consideration of the interests that parties would achieve through the duration. Therefore, the main idea of the proposed analysis is that a careful investigation only of the contractual interests of the parties, aside from any abstract and typological approach, can offer a convincing way of solving problems about the still discussed nature of long-term contracts.
\end{abstract}

Keywords: Long-Term Contracts - Functional Divisibility of the Relationship - Durable Concrete Cause - Dynamic Consideration of Interests - Duration as Applicative Category

\section{El marco teórico-dogmático de partida}

La sociedad moderna impone cada vez con mayor frecuencia la adopción de contratos destinados a la satisfacción de necesidades duraderas o recurrentes. En la actualidad las relaciones de duración se realizan en el ámbito social o económico en virtud de mecanismos cada vez más complejos que caracterizan el sistema de las relaciones que median entre individuos, organizaciones y empresas.

Debido a que la amplia difusión de relaciones diacrónicas impacta inevitablemente nuestro ordenamiento jurídico: a pesar de ello, una consciencia como tal no encuentra una amplia respuesta en la doctrina civilista contemporánea. En efecto, esta última ha terminado por estudiar marginalmente el tema de las relaciones jurídicas de duración y por relegar hasta ahora su análisis dentro de los exiguos espacios dedicados, por lo general, al estudio del derecho de las obligaciones.

El punto de partida está representado por el paradigma de las obligaciones de ejecución continuada o periódica, cuyo fundamento normativo se encuentra en el código civil y en la legislación especial más reciente (por ejemplo, pero no solo, en la legislación sobre los contratos entre consumidores y profesionales, así como en la relativa a los contratos entre empresas).

En consecuencia, debemos atribuir cierta relevancia a la comparación: bajo este aspecto, realizaremos breves referencias al contrat à exécution successive en el nuevo código civil de Quebec de 1994; a los contrats successifs en la doctrina francesa; a los contratos duraderos y a la obligación duradera en la literatura española; a la experiencia estadounidense, con toda una variedad terminológica que va desde los continuing contracts, a los severableo divisible contracts (en contraposición a los entire contracts), y a los installment contracts o output y requirements contracts; $\mathrm{y}$, por último, a la teoría de los relational contracts (contratos relaciones) en el contexto de los contratos incompletos (o que completan su contenido en base a las condiciones de determinado mercado, incluso de manera sobrevenida en relación a la celebración originaria).

Sin embargo, la reflexión sobre las obligaciones de duración presenta orígenes antiguos y se remonta a la especulación de los juristas alemanes que se extiende al período de la modernización ${ }^{(1)}$. La teorización alemana ha

(1) La acentuada tendencia de la experiencia alemana hacia las clasificaciones identifica a los Dauerschuldverhältnisseo LangfristigeVerträge como una de las clases (la principal) de los Dauerrechtsverhältnisse: para mayores referencias sobre el punto véase Otto Von Gierke, "Dauernde Schuldverhältnisse," Jher. Jahrb., vol. 64 (1914): 407 y siguientes. Reproduce la misma clasificación D. Wostney, Die rechtliche Bedeutung des wichtigen Grundes für den Bestand von Dauerrechtsverhältnissen, Diss. Frankfurt (1936): 7 y siguientes; Günther Beitzke, "Nichtigkeit, Auflösung und Umgestaltung 
Francesco Longobucco

representado un modelo importante para la doctrina civilista italiana, la cual terminó por reproponerla, con adaptaciones parciales, en nuestro sistema.

Un primer corolario de dicha reproposición es la de la referibilidad de las relaciones diacrónicas, por parte de nuestra doctrina, exclusivamente al contexto de las obligaciones ${ }^{(2)}$. En segundo lugar, el de la transición in subiecta materia del contrato de duración a la relación obligatoria de duración, esto en el marco del fenómeno más amplio de escisión entre vicisitudes del acto y vicisitudes de la relación (con referencia bien a los contratos de ejecución continuada o periódica, argumento ex artículos 1360, segundo párrafo, y 1458, primer párrafo, último párrafo, código civil italiano, bien a los contratos de ejecución diferida, -artículo 1467 código civil italiano, bien incluso a los contratos con objeto futuro, artículo 1348 código civil italiano) (Donisi, 1967). Por último, en tercer lugar, el de la elaboración (también en nuestra literatura) del supuesto de hecho de la obligación negativa como paradigma característico de obligación de duración ${ }^{(3)}$.

En el contexto de esta tendencia a estudiar el fenómeno de la duración, al igual que la doctrina alemana, únicamente dentro del sistema del derecho de las obligaciones se desarrolló, en nuestros juristas, el debate sobre la pluralidad o unidad de causas, obligaciones y contratos en la relación de duración. De esta forma, algunos acogieron la teoría de la obligación unitaria asistida por una pluralidad de actos ejecutivos (prestaciones) a la luz del esquema de la relación fundamental (por ejemplo, en materia de contratos de renta) (Lener 1967).

Del otro lado del fundamento unitario o pluralista de la relación de duración, la relevancia del tiempo sobre la estructura de las relaciones bajo análisis fue asociada principalmente a la teoría de la solutio reiterada como condicio sine qua non para la calificación del caso como obligación de duración: esta es la posición de Oppo y de Stolfi( ${ }^{(4)}$, allí donde la opinión de la doctrina alemana se muestra más amplia y destinado a incluir en la categoría objeto de estudio, no solo a las relaciones que generan una

von Dauerrechtsverhältnissen,"Schloss Bleckede an der Elbe(1948): 4. Véase, anteriormente, Bernhard Windscheid, "Die Voraussetzung," AcP, 78 (1892): 161 (el que, no obstante, es precedido por un estudio titulado Die Lehre des romischen Rechts von der Vorassetzung, Düsserdolf, 1850, al que hace referencia Francesco Macario, Vertragsanpassung und Neuverhandlungsplifcht in italienschen und deutschen Recht. EineRechtsvergleichendeDarstellungimLichte des europdischen Vertragsrecht, texto de la ponencia desarrollada en Catania, el 6 de octubre de 2006, 69). Por otra parte, en la construcción de la categoría, la literatura alemana muestra, respecto a la italiana, un margen de maniobra más amplio sobre el plano exegético, ya que en Alemania las relaciones de duración no tuvieron un reflejo normativo y, por tanto, durante mucho tiempo, el campo de análisis solo fue ocupado por doctrina y jurisprudencia.

(2) Se alude al famoso estudio de Giorgio Oppo, I contratti di durata, publicado en tres partes en Rivista del diritto commerciale e del diritto generale de lle obbligazioni(1943, I): 142 y siguientes, 227 y siguientes y, (1944): 17 y siguientes, al que se referirá en las citas (y ahora también en Obbligazioni e negozio giuridico. Scrittigiuridici [III, Padua, 1992]:200 y siguientes), el que representa aún hoy el punto de referencia de la literatura italiana sobre la materia. Véase además el texto de Luigi Devoto, L'obbligazione a esecuzione continuata(Padua, 1943); anteriormente Luigi Devoto, "Appunti per una definizione delle obbligazioni a esecuzione continuata,"Rivista di diritto civile(1942): 295 y siguientes, en donde se define al argumento bajo análisis como antiguo.

(3) Leornado Coviello Jr., L'obbligazione negativa (Contributo a lla teoría de lle obbligazioni), I y II, Nápoles, 1931, y II, Nápoles, 1934, aquí y allá .Observa este último autor, en la página 211, que el plazo, en las obligaciones negativas, tiene carácter de esencialidad (la doctrina alemana habla de negocio a plazo fijo, o de absolutes Fixgeschäft). El autor sostiene, además en la página 214 y siguientes, que la obligación de ejecución continuada no puede ser positiva, presentándose (siempre y únicamente) bajo la forma de una omisión continuada (cursivo original). Recientemente, el tema ha sido retomado por Stefano Deplano, Le obbligazioninegative(Nápoles, 2014), 82 y siguientes.

(4) Giorgio Oppo, I contratti di durata, 156. Por otra parte, ya en otro lugar, el distinguido autor se basaba en el dato estructural del cumplimiento en función distintiva respecto a otras relaciones jurídicas: pensemos en el conocido trabajo sobre Adempimento e liberalità, (1947), reimpresión 1980, Camerino, aquí y allá, en donde el cumplimiento, de estructura contractual con causa 
Relaciones de duración y divisibilidad del reglamento contractual: apuntes para una reconceptualización

Long-term contracts and divisibility of contractual regulation: notes for a reconceptualization

prestación duradera, sino también aquellas que fundan, más genéricamente, un vínculo que dura en el tiempo (dauernde Bindung). Por tanto, el elemento caracterizador, en nuestra doctrina, de la obligación de duración es esencialmente el tòpos estructural del cumplimiento reiterado de la prestación en el tiempo, destinado a incidir también en las vicisitudes de la obligación de ejecución continuada y periódica (novación, compensación, remisión, confusión, imposibilidad sobrevenida y prescripción), de modo que el efecto extintivo ha sido referido, según los casos, a los actos de cumplimiento individuales, según el enfoque de la extinción progresiva( ${ }^{(5)}$.

Por tanto, coherentemente, se ha excluido del catálogo de las relaciones de duración, por ejemplo, a la hipótesis de contrato de obra (de prestación instantánea), reconducida más bien a la categoría intermedia de contrato de ejecución diferida ${ }^{(6)}$, y a la de los contratos de ejecución fraccionada, caracterizadas por el interés instantáneo del acreedor.

Finalmente, aquello que da forma a la obligación de duración, según nuestros juristas, es la causa (abstracta) de duración, de modo que emerge además la importancia cuasi causal (tal como lo define Oppo) del tiempo sobre la prestación (Di Majo 2004, 612). Con el consecuente debate ya referido sobre la unidad o pluralidad de causas de los actos ejecutivos individuales.

\section{Hacia una reconsideración de las relaciones de duración: de la causa temporis obligationis abstracta a la divisibilidad funcional del reglamento}

Si este es el marco de partida, esbozado sintéticamente, de la communis opinio tradicional, el presente estudio parte de la perspectiva de desarrollar, metodológicamente, una deconstrucción y reconstrucción evolutiva de las relaciones diacrónicas centrada no tanto en el elemento estructural de la actividad solutoria reiterada, sino en la función de duración del negocio ${ }^{(7)}$. Ello en base a la idea de que a la consideración de la causa temporis obligationis abstracta se debe añadir el análisis del reglamento concreto, objeto y contenido del contrato, con el fin de analizar la divisibilidad funcional de las prestaciones individuales, una en relación a la otra y frente a las futuras. Por tanto, es en el específico contenido contractual, variable según el caso, que debe identificarse el

solvendi, se eleva a carácter discriminador entre liberalidad, donación y obligación. M. STOLFI, "L'appalto e i contratti di durata," Studi in onore di Giuseppe Chiarelli (Milán, 1973-1974) IV: 4202, para quien "hay extinción progresiva de la obligación, dado que el propio cumplimiento es progresivo, en la medida en que la prestación deba ser cumplida durante todo un período de tiempo, de manera continua y en todo momento" ; a dichas conclusiones, no obstante, Stolfi ya había llegado en 1940, cuando como conclusión del estudio titulado Appunticritici sui contratti di durata, en Studi in onore di B. Scorza (Roma, 1940): 864, sostenía que en las relación de ejecución continuada "se tiene la reproducción de un mismo resultado contractual completo y definitivo de todo período de tiempo individual, [y] los pagos realizados en los vencimientos individuales constituyen el cumplimiento total de diversas obligaciones autónomas, cuyo surgimiento está determinado".

(5) Luigi Devoto, L'obbligazione a esecuzione continuata, 368 (cursiva añadido). Observa que la relación de duración "se eleva a categoría autónoma de obligación, estando caracterizada por una tipología propia de causas extintivas”, Adolfo Di Majo, "Delleobbligazioni in generale, sub artículos 1173-1320," Commentario del codice civile Scialoja e Branca (Boloña-Roma, 1988): 86.

(6) Véase Giorgio Oppo, I contratti di durata, 164 y siguientes; Domenico Rubinoy Giovanni ludica,"Dell'appalto, sub artt. 1655-1677,"Commentario del códice civile Scialoja e Branca, 3a ed.(Boloña-Roma, 1992), 182 y siguientes.

(7) Hemos expresado esta opinión en Francesco Longobucco, Rapporti di durata e divisibilità del regolamento contrattuale (Nápoles, 2012), 71 y siguientes. (con recensión de Francesco Astoneen Rassegna didiritto civile (2014), 1389 y siguientes.; y de Mauro Grondona, en Andrea D’Angelo y Vincenzo Roppo, Annuario del contratto 2012(Turín, 2013), 309 y siguientes), al que permítasenos reenviar. Recientemente, la misma posición ha sido referida por Antonino Cataudella, I contratti. Parte generale, $4^{\mathrm{a}}$ ed.(Turín), 260. Referencia también en Fabio Addis, II mutamento delle condizioni patrimoniali dei contraenti (Milán, 2013), 147, nota 44; Fabio Macioce, Rendita vitalizia e perpetua, bajo el cuidado de Vincenzo Roppo, Trattato dei contratti (Milán, 2014), 112, nota 6. 


\section{Francesco Longobucco}

"instrumento cognitivo" (Orlando 1997, 35) de la categoría de las relaciones de duración. Con una incidencia, actualmente cada vez más necesaria, del recurso a una interpretación imprescindiblemente integrada del régimen de las obligaciones y del derecho de los contratos(8).

De esto resulta que, en una perspectiva de carácter funcional en el enfoque a las relaciones diacrónicas, ya no se justifican algunas exclusiones automáticas, que ocurrían en el pasado, del catálogo de la categoría bajo análisis: pensemos en las relaciones reales o caracterizados por elementos atípicos de realidad, en los contratos de seguro, de fianza, de mutuo, en la locatio operis. Como se observará a continuación, efectivamente, el principio técnico más moderno de la variabilidad de la estructura también impone verificar si las distintas estructuras de la obligación (relaciones obligatorias strictu sensu entendidas) pueden quedar sometidas a la aplicación del régimen de los contratos de duración ${ }^{(9)}$.

En consecuencia, al esbozar los caracteres de la relación de duración, es necesario confirmar la convicción preliminar de que la qualitas de la duración es el fruto de una atenta interpretación de los intereses que se hallan en el reglamento, lo que implica evidentemente la superación de la causa temporis obligationis traslaticia y abstracta a través de la recuperación de la obligación al negocio-fuente.

En este marco, lo que determina la inclusión o no del negocio en la categoría unificadora de la relación de duración (con consecuente aplicación de su propio régimen) es, según nuestra opinión, la verificación en orden a la escindibilidad funcional de las prestaciones en el programa global de las partes. Este no es predeterminable a priori y, como se observaba, de alguna forma presupone una consideración imprescindible del reglamento concreto. Nuestra doctrina parece tender a acoger una perspectiva de sistema como tal cuando, escapando de toda clasificación, siempre igual a sí misma, del contrato de suministro como contrato de duración, analiza el caso del suministro no de duración, o de la prestación periódica en la que los descuentos de los precios de los bienes y servicios son realizados en función de los montos de las cantidades globales, de modo que la cuantía del precio de los pagos ya efectuados no expresa el negocio global. El reglamento contractual es, en este caso, inescindible y las distintas prestaciones son interdependientes entre sí en el negocio general. Como resultado, el interés global a la programación (Orlando 1997, 55 y siguientes) excluye, en esta hipótesis concreta, la calificación de relación de duración del supuesto de hecho.

Lo que debemos repensar es precisamente la teoría tradicional de la unidad de la relación jurídica, ya que el mismo contrato de suministro será un tipo negocial referible a la categoría de las relaciones de duración, cuando la relación sea funcionalmente escindible en diversas prestaciones autónomas, y cuando no, no podrá incluirse en aquella categoría, ya que realiza una operación económica fundamentalmente unitaria (si bien indudablemente caracterizada por la duración legal de los efectos: condicio, no obstante, insuficiente para legitimar dicha inclusión) ${ }^{(10)}$. En consecuencia, el juicio de inclusión en el catálogo de las relaciones de duración no puede imputarse a la mera reconducibilidad del caso a determinado tipo contractual, sino, de manera mucho más compleja, deriva únicamente de un atento análisis ex post de los intereses concretos de las partes y de la fraccionabilidad en el tiempo del resultado perseguido.

(8) Pietro Perlingieri, Ildiritto civile nellalegalitàcostituzionalesecondoil sistema italo-comunitario dellefonti (Nápoles, 2006), 849 y siguientes, quien estigmatiza "la consciencia difundida [a no considerar] la historicidad y la consecuente relativización de las categorías jurídica, [y, por tanto, a considerar] a la obligación como [una] categoría ahistórica 'siempre igual a sí misma', 'neutral', elaborada tomando el mínimo común denominador de las distintas obligaciones y reduciendo las diversidades a aspectos descriptivos y externos al concepto, espurios respecto a él".

(9) Infra nota 3.

(10) Permítasenos reenviar a Francesco Longobucco, Rapporti di durata e divisibilità del regolamento contrattuale, 71 y siguientes. 
Relaciones de duración y divisibilidad del reglamento contractual: apuntes para una reconceptualización

Long-term contracts and divisibility of contractual regulation: notes for a reconceptualization

En este contexto puede resultar de ayuda (aun cuando no sea autosuficiente) el Análisis Económico del Derecho (en adelante, "AED") a través del cual se estudian, por ejemplo, los relational contracts en los Estados Unidos. En efecto, una reciente elaboración, en el contexto del AED y en el intento de identificar un objetivo concreto (función práctica y, por ende, no meramente abstracta) de las relaciones diacrónicas, tiene a reconstruir un derecho eficiente de los contratos de empresa de duración, considerando a las relaciones de duración en la perspectiva económica, como instrumento de organización y regulación de un segmento del mercado, según las razones que llevan a su adopción (Granieri 2007): con la consecuencia de que el análisis de las normas jurídicas de las relaciones de duración (sobre el desistimiento, la resolución y la excesiva onerosidad sobrevenida) será conducido, a la luz de la opinión bajo análisis, con prevalente atención a las dinámicas internas a la dimensión ejecutiva del contrato, haciendo referencia a distintos parámetros como la funcionalidad a la organización industrial, la dependencia de las (e incidencia sobre las) condiciones de mercado, la relevancia de los costos de transacción en el momento post-formativo, el interés de las partes a la estabilidad y a la flexibilidad de la relación y el fuerte elemento relacional (Granieri 2007, 225).

\section{3. (Continúa) Aplicaciones de la escindibilidad funcional de las prestaciones. Teoría de las vicisitudes de las relaciones de duración}

Por otra parte, del análisis de la divisibilidad del reglamento a la luz de los intereses deducidos en este, ya sugerida en el enfoque moderno de la doctrina al tema de la resolución parcial y de la resolución por incumplimiento/por excesiva onerosidad sobrevenida de los contratos de duración, se obtienen confirmaciones del carácter dirimente de la escindibilidad funcional de las prestaciones en las relaciones de duración. Por ejemplo, la justificación de la irretroactividad de la resolución ex artículo 1458, primer párrafo, último párrafo, código civil italiano, no radica en la imposibilidad de repetir la prestación ya ejecutada, ni en la teoría de la causa autónoma de las armadas individuales, ni en la ratio sancionatoria del remedio resolutorio, ni en la naturaleza y estructura especial de la obligación (y del cumplimiento relativo) deducida en la relación (Auletta 1942, 283), ya que ninguna connotación objetiva y estructural caracteriza la presunta unidad y escindibilidad de un negocio(11). Por el contrario, el abandono progresivo del criterio temporal en la investigación sobre la divisibilidad concreta del reglamento ${ }^{(12)}$ y la adopción del criterio funcional (vinculado al resultado a realizar) actualmente no solo alimenta el debate sobre la divisibilidad de las relaciones de duración, sino también respecto a la dinámica jurídica que caracteriza a las hipótesis más complejas, como la de la vinculación ${ }^{(13)}$ o la del fraccionamiento negocial(14).

Por el contrario, nuestra jurisprudencia de legitimidad aún parece estar fuertemente influenciada por la tradición del siglo pasado o por el elemento meramente estructural del cumplimiento reiterado de la prestación (a todo cumplimiento corresponde un segmento ex se abstractamente autónomo de la relación escindible de los otros cumplimientos). Dicha jurisprudencia sigue aplicando a priori el

(11) Son aceptables las observaciones en dicho sentido de Alberico Gentili, La risoluzioneparziale, (Nápoles,1990), 33.

(12) Ya autorizadamente sugerido por Raffaele Cicala, Concetto di divisibilità e di indivisibilità dell'obbligazione, 1953, (2a reimpresión Nápoles, 2010)aquí y allá; Raffaele Cicala, "Divisibilità e indivisibilità dell'obbligazione,"Rivista di diritto civile I (1965): 453 y siguientes; Raffaele Cicala, "Obbligazione divisibile e indivisibile," Novissimo digesto italiano XI (Turín, 1965): 636 y siguientes.

(13) Pietro Perlingieri, Nuovi profili del contratto, en Pietro Perlingieri, Il diritto dei contratti tra persona e mercato (Nápoles, 2003), 425 y siguientes. Véase, anteriormente en sentido conforme, Michele Giorgianni, Negozi giuridici collegati, ahora en Scritti minori (Nápoles, 1988), 1 y siguientes; Alberico Gentili, La risoluzione parziale, 187 y siguientes.

(14) Cónfer el reciente estudio de Andrea María Azzaro, Contratto e negozionel "frazionamento" del rapporto giuridico (Turín, 2009), 165 y siguientes. 
Francesco Longobucco

principio de la retroactividad débil (ex nunc) en materia de excepción de incumplimiento ${ }^{(15)}$ y de relaciones entre demanda de resolución y demanda de cumplimiento ${ }^{(16)}$. En cambio, la cuestión merece ser analizada a la luz, precisamente, del criterio funcional elegido. En esta clave, se debe superar el principio tradicional de reciprocidad específica entre prestación incumplida y excepción dilatoria ex artículo 1460 código civil italiano, con consiguiente valorización de la excepción en el contexto funcional de toda la relación diacrónica(17), así como será el acreedor quien deba optar por la resolución total o parcial de la relación, exclusivamente en base a los intereses que él pretenda perseguir.

El criterio funcional caracteriza, además, una reconstrucción más actual de las vicisitudes de las relaciones obligatorias de duración, centrada ya no en el dato estructural de la extinción progresiva y de la modalidad ejecutiva elegida, o en el lapso de tiempo más o menos largo transcurrido (more del deudor), sino más bien en base: (i) al criterio de la autonomía y separabilidad funcional de las prestaciones; (ii) así como al juicio del apreciable interés de las partes a la útil continuación de la relación ${ }^{(18)}$. Con adopción no de una teoría de las vicisitudes de las relaciones de duración elaborada ratione temporis (es decir, a razón del único dato de la extensión temporal de la relación), sino inspirada principalmente en los intereses de las partes (acreedor y deudor) convenientemente ponderados entre sí (así en materia de mora debendi, inexigibilidad de la prestación, imposibilidad sobrevenida total y parcial, nulidad parcial, vicisitudes relativas a obligaciones negativas de duración, todas inspirada en la utilidad del acreedor y no tanto en el cumplimiento progresivo de las prestaciones) $)^{(19)}$.

(15) Cónfer Casación No. 7550, del 15 de mayo de 2012, que hace referencia a Casación No. 12978, del 6 de setiembre de 2002 (en Repertorio de II Foro Italiano. Legislazione, bibliografia, giurisprudenza, 2002, voz Contratto in genere, No. 507), según la cual, "en los contratos de ejecución continuada o periódica, el sinalagma, a cuya tutela está predispuesto el remedio contenido en el artículo 1460 código civil italiano, debe ser considerado separadamente para la entrega de cada artículo individual, y el equilibrio sinalagmático es actuado constantemente entre prestación y contraprestación. De modo que, la adaptación de dicho principio general a la peculiaridad de los contratos de ejecución continuada o periódica, implica que, para dichos tipos de contrato, la excepción de incumplimiento puede ser hecha valer útilmente solo si atiene a la prestación de referencia en relación a la contraprestación exigida a exceptuante" (cursiva agregada).

(16) Cónfer Casación No. 26199, del 6 de diciembre de 2011, en Contratti, 2012, 121 y siguientes, para la observación según la cual "el valor abdicativo de la demanda de resolución de un contrato de ejecución continuada o periódica, respecto a la intimación de cumplimiento, debe ser circunscrito a aquella única parte de la relación para la que es posible una elección, precisamente entre resolución y cumplimiento, según el artículo 1453, segundo párrafo, código civil italiano; mientras que respecto a las prestaciones ya ejecutadas, el acreedor conserva el derecho de recibir la contraprestación" (cursiva agregada).

(17) Alberico Gentili, La risoluzione parziale, 253, quien observa que, al igual que la resolución (parcial), el remedio dilatorio de la excepción ex artículo 1460 código civil italiano, también puede comprender casos en los que, en excepción al principio de reciprocidad específica, el incumplimiento de la prestación hace desaparecer integralmente el interés del acreedor. El autor refiere, además, la siguiente jurisprudencia conforme: Casación No. 1119, del 23 de febrero de 1982, en Repertorio de II Foro Italiano. Legislazione, bibliografia, giurisprudenza I(1982), c. 1607 (el incumplimiento del promitente vendedor del deber de garantizar la inexistencia de hipotecas, prendas y secuestros puede justificar el rechazo total de cumplir); Casación No. 1275, del 11 de mayo de 1973, en Repertorio generale de lla giurisprudenza italiana I(1973, 1), c. 1654 (el incumplimiento por parte del arrendador de las obras de mantenimiento extraordinarias necesarias para la conservación de la cosa arrendada en estado de servir al uso, puede justificar la suspensión pro futuro del pago del canon) (Alberico Gentili, La risoluzione parziale, 252, nota 94).

(18) Para mayores desarrollos permítasenos reenviar, una vez más, a Francesco Longobucco, Rapporti di durata e divisibilità del regolamento contrattuale, 110 y siguientes.

(19) Francesco Longobucco, Rapporti di durata e divisibilità del regolamento contrattuale.Entonces, en este contexto, no parece tener mucha utilidad el intento de diversificar, dentro de una categoría tradicionalmente unitaria como la de las obligaciones de ejecución continuada y periódica, el estatuto normativo, por una parte, de las obligaciones de ejecución continuada y, por el otro, de las obligaciones de ejecución periódica, respectivamente. Dicho intento parte de la idea de que la distinta estructura de ambas tipologías de obligaciones en cuestión conduzca a un régimen diferenciado (según se trate de 


\author{
Relaciones de duración y divisibilidad del reglamento contractual: \\ apuntes para una reconceptualización \\ Long-term contracts and divisibility of contractual regulation: \\ notes for a reconceptualization
}

El paradigma de la divisibilidad funcional refuerza además la perspectiva de la superación de la lógica del tipo contractual(20): por consiguiente, es aceptable aquella tendencia a aplicar, en vía extensiva o analógica, poco importa, el régimen de las relaciones de duración incluso a hipótesis tradicionalmente excluidas del catálogo de las obligaciones duraderas (el ejemplo paradigmático es el de la venta de ejecución fraccionada)(21). Ulteriores confirmaciones respecto a la divisibilidad funcional del reglamento negocial se obtienen de la Convención de Viena sobre la Compraventa Internacional de Mercaderías (en español, artículo 73.3), en donde se halla de iure condito precisamente una referencia expresa a la (eventual) interdependencia de las prestaciones individuales.

\section{4. (Continúa) La relación de duración como categoría aplicativa y producto de la interpretación del caso concreto}

Como resultado, podemos desarrollar algunas primeras conclusiones. En realidad, las relaciones de duración constituyen una categoría aplicativa que se identifica por algunos elementos esenciales en la reconceptualización hasta ahora desarrollada: (i) la función concreta de duración, entendida como satisfacción de los intereses de las partes únicamente a través del tiempo(22), y (ii) la autonomía de las prestaciones individuales deducida del conjunto de intereses del reglamento (argumento del criterio de la retroactividad débil normativamente establecido por el artículo 1458, primer párrafo, último párrafo, código civil italiano). De esta forma, la duración es un estatuto normativo especial de las relaciones jurídicas, inspirado principalmente en el principio de conservación de los efectos ya producidos.

La categoría de las relaciones de duración es, en otros términos, exclusivamente un posterius de la interpretación en función aplicativa,

obligaciones continuadas o periódicas) de las vicisitudes de la relación obligatoria, aspecto, este último que en realidad constituye una de las consecuencias menos investigadas en nuestra literatura (así Roberto Lattanzi, I rapporti di durata, edición provisoria(Milán, 1996), en español, 137 y siguientes).

(20) De lo cual he tratado de dar cuenta con relación a la hipótesis del contrato de prestación de servicios, la cual ha legitimado un extenso debate en mérito al régimen aplicable a ella en abstracto. El problema no parece ser correctamente planteado por la doctrina. "De hecho, la excesiva referencia a la estructura tipológica del supuesto de hecho corre el riesgo de falsar los resultados a los que el intérprete correctamente debería llegar. En sentido estricto, es innegable que en los contratos de prestación de servicios se pueda incluir el aspecto de la duración (periodicidad o continuidad de la prestación) y que en los hechos, a menudo, lo sea. En consecuencia, este aspecto no puede no incidir sobre la función (de duración) y, por tanto, sobre la identificación de la normativa aplicable al caso concreto. La relevancia del dato de que las prestaciones tienen carácter duradero y no instantáneo, conduce al intérprete a aplicar, en el respeto de los intereses perseguidos en el negocio, el peculiar estatuto de las relaciones de duración (artículos 1360, 13373, 1458, primer párrafo, último párrafo, y 1467 código civil italiano, así como el mismo régimen del suministro), donde las prestaciones de hacer son funcionalmente escindibles entre sí. Igualmente, junto a dicho régimen encontrará aplicación el del contrato de obra: esto sin atribuir preeminencia a uno de los tipos contractuales, sino según la técnica de la "disposición conjunta" (argumento, además, ex artículos. 1570 y 1677 código civil italiano)" (Francesco Longobucco, Rapporti di durata e divisibilità del regolamento contrattuale, 130,128 y siguientes para los términos del debate).

(21) De hecho, lo que cuenta no es aclarar el modo "en que se ha previsto el fraccionamiento de las entregas" (una entrega fraccionada puede haber sido juzgada convencionalmente como infraccionable: Gerardo Santini, I contratti "divisibili", en Rivista didiritto civile I (1982): 13 y siguientes). Más bien, es útil aclarar las modalidades concretas de toda hipótesis individual, analizar si las partes han valorado la relación diacrónica como divisible o indivisible (Cónfer Angelo De Martini, Profilidella vendita commerciale e del contratto estimatorio (Milán, 1950), 279, según quien es necesario evitar, "afirmaciones axiomáticas a priori, en los casos de difícil diferenciación práctica").

(22) El estudio de Angelo Barba, Il contratto di sponsorizzazione e somministrazione di servizi, en bajo el cuidado de Roberto Bocchini, I contratti di sponsorizzazione di servizi(Turín, 2006), 905 y siguientes, se muestra de acuerdo con este planteamiento, quien reconstruye la duración como acto llamado "a realizar un interés decisivo de la función de autonomía 
Francesco Longobucco

según una valorización más moderna de los casos concretos, con el efecto de que el carácter de la escindibilidad funcional de las prestaciones en el tiempo, como dato calificador de las relaciones bajo análisis, debe ser asumido como un "deber ser" (Gentili 1990, 181), dotado de sentido solo como criterio de elección funcional y no como dato estructural. Por tanto, la escindibilidad no es criterio, sino solo resultado de una elección interpretativa. El régimen de las relaciones de duración es, de hecho, un instrumento dirigido esencialmente a componer, con la ayuda, ineludible, del intérprete, un conflicto de intereses de tipo "prospectivo" (Femia 1996, 454), es decir, posterior a la celebración del acto, porque se manifiesta en la fase de cumplimiento de las prestaciones divisibles entre sí en el resultado programado por las partes (y, por tanto, directamente in executiviis en la relación-reglamento).

En esta perspectiva, suscitan algunas perplejidades aquellas opiniones que atribuyen la calificación de relación de duración también a las relaciones funcionalmente inescindibles ${ }^{(23)}$, ya que, viceversa, en el caso de relaciones no divisibles (id est: no de duración), debería revivir el estatuto normativo ordinario (resolución y retroactividad fuerte ex tunc) ${ }^{(24)}$.

\section{Función concreta de duración y variabilidad de las estructuras negociales}

La función concreta de duración, en la que se centra la identificación de la categoría bajo análisis, debe ser vinculada al moderno principio técnico de la variabilidad de la estructura negocial, en base a la idea de que la realización del interés duradero pueda cumplirse a través de diferentes situaciones jurídicas subjetivas activas y pasivas, derechos subjetivos, expectativas jurídicas, deberes y sujeciones, vinculadas de manera diversa en la relación jurídica ${ }^{(25)}$ : con la consecuencia de una posible extensión de la categoría de las relaciones de duración.

En este contexto se inserta, por ejemplo, la reconducción a la categoría de las relaciones de duración, ya acogida por alguna doctrina, del contrato de mutuo(26) (por otra parte, la jurisprudencia también ha aplicado el régimen

privada y absolutamente particular" (página 938); en este contexto, específicamente, "la duración de la acción útil, en la medida que esté contextualizada, revela la función que ordena y organiza soluciones normativas a tipos de problemas, vale decir, determina el contenido de sentido de la compatibilidad que permite la organización del tipo normativo y, con esta, la aplicación directa de la norma" (página 946) (cursiva agregada).

(23) Véase las conclusiones de Stefano Pagliantini, La risoluzione dei contratti di durata (Milán: Guiffré, 2006) aquí y allá.

(24) Es el sostén de nuestra investigación conducida en Rapporti di durata, 126 y siguientes, al que permítasenos reenviar. Con un corte parcialmente distinto, reconduce la categoría de la duración a la de la divisibilidad, la obra contemporánea de Alessandro Gnani, I/ contratto divisibile(Nápoles: Jovene, 2012), 149 y siguientes.

(25) La observación, por otra parte, absolutamente aceptable sobre el plano metodológico y aplicativo, caracteriza el reciente análisis de Angelo Luminoso, IIrapporto di durata, en Rivista di diritto civile (2010): 525 y siguientes. Este se centra en la relevancia del interés (duradero) sobre la estructura negocial, que puede ser la más diversa: Cónfer Pietro Perlingieri, Ildiritto civile nellalegalitàcostituzionalesecondoil sistema italo-comunitario dellefonti, 604 y siguientes, para la observación de que "una misma función puede realizarse mediante una pluralidad de estructuras. La variabilidad de la estructura negocial depende de la función y de las relaciones sobre las que el acto incide" (cursiva agregada). Véase además, Pietro Perlingieri y Pasquale Femia, Nozioniintroduttive e principifondamentali del diritto civile, $2^{a}$ ed., con la colaboración de Loredana Tullio, 2 (Nápoles: Edizioni Scientificheltaliane, 2004), 101 y siguientes; Rosario Nicoló, Ilriconoscimento e la transazionenel problema dellarinnovazione del negozio e dellanovazionedell'obbligazione, (1934-1935), en Raccolta di scritti, vol. 1(Milán: Giufrré, 1980), 416, pero tomando en consideración una terminología menos cierta, donde función designa un objetivo genérico, una causa común, una suerte de esquema clasificatorio de una pluralidad de actos de eficacia diversa, y estructura tiene el significado singular de eficacia, contenido.

(26) Cónfer en especial, G. Giampiccolo, Comodato. Mutuo, en Trattatodi diritto civile Grosso y Santoro Passerelli (Milán, 1972), 69 y siguientes; E. Simonetto, en Enciclopedia giuridica Treccani, vol. 20 (Roma, 1990), 6 y siguientes. 


\section{Relaciones de duración y divisibilidad del reglamento contractual: apuntes para una reconceptualización Long-term contracts and divisibility of contractual regulation: notes for a reconceptualization}

de los contratos de duración al caso del mutuo inmobiliario(27), del contrato de seguro(28), del contrato de arrendamiento, de comodato y anticresis ${ }^{(29)}$. Respecto a estos contratos, la función negocial de satisfacer el interés duradero del acreedor es realizada mediante una atribución patrimonial (o una prestación en sentido amplio) distinta del cumplimiento en sentido clásico. También el esquema del contrato de obra, tradicionalmente excluido del catálogo de los contratos de duración, puede, a tal efecto, realizar una función de duración, especialmente a la luz de los profundos cambios organizativos y económicos que lo han afectado y de la superación, al menos en algunas hipótesis más complejas de posible escindibilidad funcional del reglamento global, de la calificación tradicional de "contrato (instantáneo) de ejecución diferida"(30).

De esto deriva la aplicabilidad incluso en materia de contrato de obra de duración (centrado en la realización de obras complejas y autónomas entre sí) del artículo 1458, primer párrafo, último párrafo, código civil italiano, relativo a la resolución por incumplimiento y del artículo 1668 código civil italiano concerniente a la resolución parcial|(31). Además: el contrato de sociedad (o asociativo en general) da origen a una vinculación entre situaciones jurídicas subjetivas, los estatus, a las que puede reconducirse la función de duración, que solo se realiza a través de la obra de unificación y coordinación entre relaciones obligatorias individuales desarrollada por el elemento causal (concreto) del contrato(32).

De los ejemplos, solo sumariamente esbozados emergen indudables cualidades de la tendencia a reelaborar, sobre el plano

(27) Cónfer expresamente, en este sentido, Corte di cassazione, sezioni unite. No. 12639, del 19 de mayo de 2008, en Giustizia civile, vol. 1 (2008): 1391.

(28) Antigono Donati, Trattato del diritto delle assicurazioni private, vol 2 (Milán: Guifrré, 1954), 43 y siguientes; Agostino Gambino, L'assicurazione nella teoria dei contratti aleatori(Milán: Guiffré, 1964),338; Angelo Luminoso, Il rapporto di durata, 529.

(29) Así concluye S. Sangiorgi, Rapporti di durata e recessoad nutum (Milán: Giuffré, 1965), 88-91, con la observación de que "el término 'arrendamiento' identifica una relación compleja que no puede ser recomprendida en calificaciones relativas a entidades simples, representadas por figuras elementales [...] como son la obligación, o el mismo 'derecho personal de goce', de manera que en relación al arrendamiento (al comodato y a la anticresis) la calificación de la posición del arrendatario, cuando quiera realizarse con el metro de relaciones jurídicas unitarias, no podrá ocurrir si no es en términos de prevalencia, tomando en cuenta el conjunto coordinado de relaciones obligatorias y de facultades que la componen". Por tanto, según este último autor (páginas 80, 84, 87, nota 159, y 90), el arrendamiento, el comodato y la anticresis "se pueden calificar como contrato de duración solo bajo la condición de tener presente que esto no implica una perfecta equiparación con los otros contratos, a los que no obstante dicha expresión también es referida. Ya que, mientras en estos últimos el cumplimiento continuado realiza casi sin residuos el interés de una de las partes, en aquellas [de arrendamiento, comodato y anticresis], por el contrario, la relación de duración no alcanza por sí misma dicho objetivo, y por el límite que encuentra no puede, por ende, ser asumido válidamente como elemento característico del contrato". [...] En efecto, en el contrato de arrendamiento, comodato y anticresis, dado que "la duración no corresponde a una obligación típica del contrato" y "no puede atribuirse a la obligación de duración un rol preeminente en la integración de los efectos del contrato de arrendamiento", se está en presencia de "incuestionables relaciones de duración, pero que resultan del goce que un sujeto tenga del bien, y no porque realizan dicho goce, [tratándose de meros] deberes integrativos primarios" (cursiva agregada). Llega a análogos resultados, más recientemente, Angelo Luminoso, Ilrapporto di durata, 512, en español nota 36.

(30) Giorgio Oppo, I contratti di durata, 164 y siguientes; Domenico Rubino y Giovanni ludica, Dell'appalto,182 y siguientes.

(31) Se lo observa en Manuale di dirittoprivato europeo, 2, bajo el cuidado de Carlo Castronovo y Salvatore Mazzamuto, (Milán, 2007),1055. Para una aplicación jurisprudencial de la resolución por incumplimiento débil (ex nunc), Cónfer Colección Arbitrale, del 21 de abril de 1983, Archiviogiuridicodelle opere pubbliche(1984): 1, según el cual "la resolución del contrato no opera ex tunc, en la medida en que no se extiende a las prestaciones ya ejecutadas, porque, al haberse desarrollado el contrato de obra a través de la ejecución de trabajos efectuados con estado de avances regulares, aprobados y pagados, debe adoptarse, en vía analógica, el régimen establecido por el artículo 1458, primer párrafo, último párrafo, código civil italiano para los contratos de ejecución continuada, y los efectos del acogimiento de la demanda se retrotraen a la fecha de su interposición". 
Francesco Longobucco

calificador, las situaciones jurídicas subjetivas de los principales contratos regulados por el código civil en una perspectiva distinta a la de la relación obligatoria tradicional y en función de la causa concreta ${ }^{(33)}$ (de duración), con resultados más coherentes sobre el plano del régimen aplicable, previa verificación de que las situaciones jurídicas subjetivas vinculadas en la relación se agoten totalmente (rectius: funcionalmente independientes) la una en relación a la otra y frente a las futuras, con referencia a un determinado arco de tiempo.

\section{Relaciones de duración atípicas, juicio de licitud y meritoriedad dinámica}

A la superación de la lógica del tipo contractual y a la valorización de la función concreta de duración se vincula el ulterior problema de la verificación de si y en qué límites la autonomía de los particulares pueda llegar a actuar una función como tal incluso fuera de las hipótesis contractuales tradicionales contenidas en el código civil( ${ }^{(34)}$. En especial, la admisibilidad de relaciones diacrónicas atípicas (incluso diferentes a las obligatorias, id est: no centradas en el cumplimiento reiterado de la prestación en el tiempo) se mueve entre límites de licitud, a menudo impuestos por los mismos plazos legales mínimos y máximos entendidos precisamente como límite preliminar de licitud de la relación duradera (límites de duración del franchising, de la multipropiedad, etcétera), y límites de meritoriedad.

Por otra parte, el juicio de meritoriedad en el supuesto de hecho de duración asume contornos de particularidad, ya que este identifica un juicio no meramente estático, sino propiamente dinámico y legitima la valoración imprescindible, en el contexto de la misma duración legal o de una duración pactada no sometida a plazos legales, de la conveniencia de perseguir, en el caso concreto, un determinado objetivo a través del tiempo(35). El artículo 1379 código civil italiano (relativo a la nulidad de la prohibición de enajenar no contenida dentro de límites de tiempo convenientes y que no responda a un interés apreciable de una de las partes) se eleva, en dicha dirección, a paradigma general del juicio de Meritoriedad en el tiempo.

Esta última norma, a modo de cláusula general, impone la necesidad de seleccionar los intereses que merecen una tutela mayor en el tiempo respecto de aquellos que merecen

(32) Clasifican a la sociedad como contrato de duración, reconociendo la existencia de un cumplimiento duradero en el tiempo, G.C.M. Rivolta, "La società come rapporto di durata,"Rivistadellasocietà (1962): 34 y siguientes; luego M. Stella Richter Jr., "Il tempo nei contratti sociali e parasociali," Rivista didiritto civile, vol 1 (2000): 663 (también en Autores Varios, La rilevanza del tempo neldirittocommerciale(Milán, 2000), 128 y siguientes).

(33) Para una reciente autorizada reconstrucción de la noción véase Cesare Massimo Bianca, "Causa concreta del contratto e dirittoeffettivo,"Rivista didiritto civile (2014): 251 y siguientes, nota 44, pie 7.

(34) Este aspecto es analizado en Rapporti di durata e divisibilità del regolamentocontrattuale, 193 y siguientes, al que permítasenos un reenvío ulterior.

(35) Un valioso análisis de la noción de Meritoriedad dinámica, rica en aspectos aplicativos, es el de Michele Ferrario Hercolani, La duratadelleobbligazioni, 4 y siguientes.

(36) En realidad, bien vistas las cosas, la norma bajo análisis (artículo 1379 código civile italiano) legitima un doble juicio de meritoriedad. En efecto, a la luz del principio de la libre circulación de los bienes, del requisito legislativo de la apreciabilidad del interés, se deduce el criterio de juicio que subordina la validez del pacto de no enajenar con el fin de perseguir utilidades en condiciones, de conformidad con la jerarquía de valores del ordenamiento, de contrabalancear el sacrificio de la libre circulación de los bienes. Estos son los objetivos superiores de la salvaguardia y promoción de la persona humana, y aquellos equivalentes, aunque instrumentales, a la eficiencia económica. Junto a este primer grado de juicio inherente a la meritoriedad de la prohibición en sí misma considerada (en sentido estático), se coloca, en la previsión legislativa, una segunda e igual ponderación (en sentido dinámico) a la que se dirige el criterio de valoración bajo el aspecto temporal de la eficacia de la prohibición, que se configura en el requisito legislativo de los convenientes límites de tiempo. El objetivo de salvaguardar la libre circulación de los bienes informa, además, las valoraciones a las que están sometidas las prohibiciones 


\section{Relaciones de duración y divisibilidad del reglamento contractual: apuntes para una reconceptualización Long-term contracts and divisibility of contractual regulation: notes for a reconceptualization}

una tutela más limitada(36). No obstante, los particulares quedan libres al elegir los plazos pactados, no siendo admisible un juicio de mérito, por parte del juez, sobre la entidad del plazo negocial de la prohibición. Alguna indicación sobre la duración congruente de la relación puede encontrarse a nivel sistemático del código civil: la duración decenal de la relación representa, de hecho, un posible ejemplo de Meritoriedad ratione temporis para las relaciones que tienen por objeto bienes que tienden a revaluarse en el tiempo; sin embargo, se pueden admitir diferentes duraciones (más largas) para las relaciones que tengan por objeto bienes que necesitan de mucho tiempo para adquirir valor, según el destino que se les da.

Entonces, la discrecionalidad y el control judicial sobre el frente de la valoración de la adecuación de la duración debe ser vinculada al tipo de interés (patrimonial o no patrimonial) a ser realizado a través de la propia duración. No hay dudas de que, en la perspectiva de la ponderación dinámica de los intereses relevantes, el interés a la tutela de la persona deba prevalecer en relación al patrimonial contrapuesto, con posibilidad de desvinculación inmediata en cualquier momento de la parte a tutelar, o con exclusión del desistimiento para toda la vida del beneficiario de la prestación.

Pensemos, por poner un ejemplo, en la duración del estatus de asociado (parametrado únicamente a la voluntad del asociado, salvo los casos de procedimiento de exclusión motivada ex artículo 24 código civil italiano), del mandato ordinario y de las relaciones gestorías intuitu personae, de la asignación de la casa familiar (cuya duración, por lo general, se extiende hasta la cesación de la destinación existencial dada al inmueble); pensemos, además, en la duración de las obligaciones que tienen por objeto un hacer o un no hacer, con especial referencia a la prestación laboral y también en dependencia de un estatus societario, así como en la exclusión del instrumento del desistimiento en el caso de renta previsional o de manutención a favor de un determinado beneficiario.

En todas estas hipótesis, la duración de la relación depende de la tutela de la persona, según el caso, merecedora de realización ${ }^{(37)}$. Por el contrario, cuando la duración de la prestación deba referirse únicamente a la tutela de la libertad económica del deudor (o de la parte en general), el interés de este último se ponderará con el interés patrimonial contrapuesto a través de la garantía de la desvinculación de la relación luego de un tiempo funcionalmente conveniente ${ }^{(38)}$.

convencionales de enajenación tipificadas por el legislador (artículos 968, tercer párrafo, 980, 1260, segundo párrafo, 235bis, 2469 código civil italiano): por tanto, se configura una relación de genusa specie respecto al fenómeno delineado por el artículo 1379 código civil italiano. De esto deriva que los requisitos del interés apreciable y de los convenientes límites temporales también operan para las figuras de pactos de no enajenar legislativamente típicas.

(37) Mayores ahondamientos sobre las distintas hipótesis indicadas en el texto se encuentran en el análisis de Michele Ferrario Hercolani, La duratadelleobbligazioni, 38 y siguientes, 41 y siguientes, 93 y siguientes, 99 y siguientes. Sobre el plano metodológico permítasenos invocar nuestra idea en base a la cual "la comparación funcional de las intenciones concretas de las partes en los contextos de duración no se limita, por tanto, a analizar la observancia de los plazos legales o la ilicitud de los pactos que excluyen mecanismos de desvinculación (como el desistimiento o el rescate), sino, en una perspectiva mucho más compleja y responsable, está predispuesta a ponderar los intereses negociales y a establecer, con mayor precisión, si determinado interés, dentro de la jerarquía actual de los valores constitucionales, merezca mayor tutela que el interés contrapuesto y en conflicto, a realizarse por vía diacrónica. En otros términos, incluso cuando el legislador establece una duración mínima o máxima de la relación y, con mayor razón, si esta no es establecida [límite de licitud necesario, mas no suficiente], no se nos podrá eximir de considerar si determinado interés pueda legitimar la duración de la relación o si, en cambio, prevalezca el interés opuesto a la salida de aquella misma relación (a través del receso o el rescate)" (Francesco Longobucco, Rapporti di durata e divisibilità del regolamento contrattuale, 208).

(38) Michele FerrarioHercolani, La duratadelleobbligazioni, 51 y siguientes, del cual se han tomado los ejemplos aplicativos indicados en el texto. 
Francesco Longobucco

Pensemos, al respecto, en la duración de la renta perpetua o vitalicia (siempre que no sea previsional o de manutención); en la duración de las condiciones que corresponden a negocios traslativos de propiedad y de aquellas que corresponden a negocios no traslativos de la propiedad de un bien material; en la duración de los vínculos de destinación y de las prohibiciones de enajenación, con especial referencia a la prelación contractual o testamentaria; en la duración en las relaciones de garantía (dejando a salvo el caso especial de la garantía relativa a obligaciones infungibles y que tienen por objeto el pago de una indemnitas en correspondencia del incumplimiento del deudor principal), en la duración de las participaciones societarias que implican una responsabilidad ilimitada del socio; en el principio general (argumento ex artículo 2125 código civil italiano) de la duración necesaria de las obligaciones negativas (dejando a salvo la hipótesis especial de una obligación de pati que puede tolerar una duración y una privación contemporánea de la facultad de liberarse, naturalmente ya muy larga, hasta de treinta años, e incluso toda la vida de un sujeto, si, por ejemplo, el negocio persigue una función previsional). Es decir, cuando entra en juego la ponderación de intereses meramente económicos y patrimoniales, en el contexto de la duración de la relación, el instrumento de ponderación se convierte en la garantía de la salida del deudor (o de la parte en general) de la propia relación.

\section{Observaciones conclusivas}

Como conclusión, pueden explicitarse algunas convicciones sobre el renovado enfoque del tema. La categoría de las obligaciones de duración es, por algunos aspectos, más restringida de lo que la opinión tradicional deja entender porque, como hemos observado anteriormente, la mera reiteración de las prestaciones durante el tiempo no es por sí misma dirimente para calificar a la relación como de duración a falta de suficiente autonomía funcional de las prestaciones individuales.

Por otros aspectos, en cambio, esta es decididamente más amplia ya que un interés concreto de las partes a la duración del vínculo y un grado de autonomía suficiente de autonomía de las prestaciones (esta última en el resultado concreto y general programado por las partes) pueden identificarse en relaciones en las que no se tiene tanto la repetición de la prestación durante el tiempo, sino más bien una prestación duradera. Pensemos en el caso del contrato de suministro, el que, si bien recientemente ha sido clasificado como un típico contrato de duración en tanto caracterizado por efectos legales de duración típicos, no se presta, en cambio, a ser calificado como tal en abstracto, es decir, prescindiendo del reglamento de intereses concreto establecido entre las partes, considerado que, sobre un plano metodológico más general, el régimen aplicable al tipo contractual no puede identificarse prescindiendo de un análisis de la operación económica efectivamente programada por los contratantes y sin además abandonar el insuficiente esquema de la unidad de la relación jurídica.

En el fondo se destaca el rechazo de fundar el análisis de las prestaciones duraderas sobre datos meramente estructurales, prestando así la debida atención a los aspectos funcionales, a los intereses concretos en juego, al análisis de los valores que deben llevar a reconocer la prevalencia de uno sobre el otro, en la convicción de fondo de que la calificación de duración no constituye un prius, sino únicamente un posterius de la interpretación para fines aplicativos.

\section{Referencias bibliográficas}

Auletta, Giuseppe. 1942. La risoluzione per inadempimento. Milán: Edizioni Scientifiche Italiane.

Donisi, Carmine. 1967. In tema di nullità sopravvenuta del negozio giuridico. Rivista trimestrale di diritto e procedura civile.

Di Majo, Adolfo. 2004. Elementiaccidentali del contratto. Al cuidado de Mario Bessone, Istituzioni di diritto privato. Turín: Giappichelli Editore. 
Relaciones de duración y divisibilidad del reglamento contractual: apuntes para una reconceptualización

Long-term contracts and divisibility of contractual regulation:

notes for a reconceptualization

Femia, Pasquale. 1996 Interessi e conflitti culturali nell'autonomia privata e nella responsabilità civile. Nápoles: Edizioni Scientifiche Italiane.

Granieri, Massimiliano. 2007. Il tempo e il contratto. Itinerario storico-comparativo sui contratti di durata. Milán: Giuffré.
Lener, Angelo. 1967. I/ rapporto di rendita perpetua. Milán: Giuffré.

Orlando, Salvatore. 1997. Contratti di durata e recesso convenzionale. Roma: Pioda. 